Rebollo-Catalán, A. Mayor-Buzon, V. y García-Pérez, R. (2017). Competencias digitales de las mujeres en el uso de las redes sociales virtuales: diferencias según perfil laboral. Revista de Investigación Educativa, 35(2), 427-444

DOI: http://dx.doi.org/10.6018/rie.35.2.270881

\title{
Competencias digitales de las mujeres en el uso de las redes sociales virtuales: diferencias según perfil laboral
}

\section{Women's digital skills in the use of social network sites: differences by employment status}

\author{
Ángeles Rebollo-Catalán,Virginia Mayor-Buzon y Rafael García-Pérez \\ Universidad de Sevilla. España
}

\section{Resumen}

Este artículo presenta resultados parciales de la investigación "las mujeres como tejedoras de las redes sociales: estrategias relacionales e inclusión digital", financiada por el Ministerio de Economía y Competitividad. El objetivo del estudio es conocer las competencias digitales de las mujeres en el uso de las redes sociales y determinar perfiles según su nivel de habilidad en las redes. Además, analizamos las diferencias en la competencia digital en función de la situación laboral de las mujeres. Con este fin, se realizó una encuesta de 1340 mujeres con diferentes perfiles en función de su situación laboral y su edad. Se aplicó una escala para medir las competencias digitales en el uso de redes sociales, que obtuvo índices óptimos de validez y fiabilidad. Los resultados muestran un nivel medio-alto de competencias en el uso de las redes sociales, siendo las mujeres profesionales y emprendedoras las que muestran competencias más avanzadas respecto a las mujeres desempleadas. Todas ellas tienen poco desarrolladas las competencias de manejo de información y creación de contenidos, lo que tiene repercusión en la formación para el empleo.

Palabras clave: habilidades; alfabetización digital; redes sociales; tecnologías de la web 2.0; mujeres.

Correspondencia: Ángeles Rebollo-Catalán, rebollo@us.es, Dpto. MIDE, Facultad de Ciencias de la Educación, Universidad de Sevilla (España), calle Pirotecnia, s/n, 41013 Sevilla. 


\begin{abstract}
This article presents partial results of the investigation "women as weavers of the social networks: relational strategies and digital inclusion", funded by the Ministry of Economy and Competitiveness. The purpose of this study is to know the digital skills of women in the use of social networks and determine the profiles according to their level of skills. In addition, we analyze differences in digital competence depending on women employment status. To this end, we conducted a survey of 1340 women with different profiles depending on their employment status and their age. We applied a scale to measure digital skills in using social networking sites, which had optimal rates of validity and reliability. The results show a medium-high level of digital skills in the use of social networks, finding that women professional and entrepreneurial show more advanced skills regarding unemployed women. All women surveyed have underdeveloped skills of information management and digital content creation, which has an impact on training for employment.
\end{abstract}

Keywords: skills; digital literacy; social networks; web 2.0 technologies; women.

\title{
Introducción
}

La presente investigación responde a uno de los objetivos principales de las políticas internacionales en materia de inclusión digital que es el vinculado con el empoderamiento de las mujeres en la Sociedad de la Información. La Agenda Digital Europea (Comisión Europea, 2013) establece como línea prioritaria la mejora de la alfabetización digital, capacitación e inclusión de las mujeres en la Sociedad de la Información. Y de acuerdo con ésta, el Plan Inclusión Digital y Empleabilidad de España (MIET, 2013) se propone entre sus objetivos aumentar la presencia y participación de las mujeres en la Sociedad de la Información a través de la formación de mujeres desempleadas, profesionales y empresarias que ya usan las tecnologías digitales con el fin de aumentar sus capacidades TIC. Esta investigación trata de documentar el nivel y tipo de competencias digitales que muestran mujeres con estos perfiles en el uso de las redes sociales, analizando posibles diferencias entre ellas cara a orientar su formación y capacitación en TIC.

Cada vez más informes alertan del peligro de exclusión digital que sufren determinados colectivos y, entre ellos, las mujeres (Braun, 2013; Dias, 2012) y los efectos en su empleabilidad (Gómez-Torres, 2016; González-Oñate, Fanjul-Peyró, \& CabezueloLorenzo, 2015; Torrent-Sellens, 2014). En concreto, Torrent-Sellens (2014) advierte de que la formación que se ofrece no logra reducir la brecha digital al no alcanzar a los colectivos menos formados y con menos competencias digitales. Luna, Mendoza y Álvarez (2015) plantean la necesidad de ofrecer aplicaciones y productos tecnológicos centrados en satisfacer las necesidades de estos colectivos. Gómez-Torres (2016) denuncia cómo la mayor parte de la investigación científica y tecnológica y de las aplicaciones que emanan de ella está centrada en el mercado más joven, por lo que concluye que difícilmente las personas mayores serán capaces de apreciar las ventajas de las actuales herramientas de formación virtual si no se sienten lo suficientemente motivadas e interesadas. 


\section{La alfabetización digital de las mujeres}

Investigaciones previas han venido mostrando que las habilidades digitales en Internet están condicionadas por el género y que la brecha digital de género persiste más allá del acceso y usos básicos de las TIC, perpetuándose en otras dimensiones como la autonomía, experiencia, habilidad y tipos de uso (Castaño, Martin, \& Vázquez, 2008; Hargittai \& Shafer, 2006). Rubio y Escofet (2013) concluyen que la experiencia de los hombres con las TIC es mayor que las mujeres, poseen antes ordenador, lo usan más, juegan más con él y lo utilizan más con finalidad de ocio, mientras que el uso de internet con fines educativos se da igual en hombres y mujeres.

Vega-Caro, Vico-Bosch y Rebollo-Catalán (2015) han revelado que las motivaciones que impulsan a las mujeres a usar las redes sociales virtuales tiene que ver con las posibilidades de fortalecer y aumentar su capital social, así como con su utilidad y aplicabilidad para ser más eficientes en las actividades que realizan. Pero la mayor parte de las investigaciones centradas en la alfabetización digital de las mujeres han mostrado que disponer de un contexto afectivo y social de apoyo, ayuda y colaboración es un predictor más significativo de la inclusión digital que la propia intensidad y variedad de uso de la tecnología por parte de la mujer (Lin, Tang, \& Kuo, 2012; Vekiri \& Chronaki, 2008), lo que hace que la vertiente social y comunicativa de uso de las tecnologías digitales, es decir, los contextos de uso, cobren especial interés en el empoderamiento de las mujeres en TIC.

Pero, otras investigadoras (Sánchez-Vadillo, Ortega, \& Vall-Llovera, 2012; Vergés, 2012) muestran que no solo son las motivaciones más utilitaristas las que producen habilidades digitales avanzadas sino que son las motivaciones por placer asociadas al ocio las que favorecen la autoinclusión digital de las mujeres, así como las actitudes más creativas y de innovación las que inciden en el aumento de habilidad digital. Recientemente Rebollo-Catalán, Vico-Bosch y García-Pérez (2015) han demostrado que son las mujeres con más habilidad digital, las que expresan mayores y más variadas inquietudes de formación en tecnologías digitales, siendo la seguridad y protección en las redes, las aplicaciones profesionales y de búsqueda activa de empleo junto con los servicios de cultura y ocio las que más se demandan.

Algunos trabajos recientes han planteado la necesidad de fijar la atención en las habilidades de creación de contenidos digitales como un indicador clave de la alfabetización digital, más allá del uso, consumo y participación (García-Ruiz, Ramírez-García, \& Rodríguez-Rosell, 2014; Lee, Chen, Li, \& Lin, 2015; Sánchez-Carrero \& Contreras-Pulido, 2012; Watson, W.R., Watson, S.L., \& Reigeluth, 2015). Pérez-Escoda y Rodríguez-Conde (2016) no solo encuentra menores puntuaciones en la creación de contenidos y resolución de problemas sino también donde mayor variabilidad se da, demostrando que, incluso, el profesorado que se autopercibe competente, no maneja estrategias en un nivel suficiente que le permita innovar. En su estudio con mujeres adultas, García-Pérez, Mayor-Buzon y Rebollo-Catalán (2016) muestran la estrecha relación entre la creación de contenidos digitales y el logro de competencias digitales avanzadas, pero también encuentran que aún menos de la mitad de las mujeres crean y mantienen webs, blogs y/o canales de YouTube, siendo una actividad poco frecuente no solo en mujeres desempleadas sino también en profesionales y empresarias. Esto confirma lo que señalan otros estudios que 
apuntan a que es en la creación de contenidos donde más persisten las brechas digitales (Navarro, 2009; Wajcman, 2012), siendo más los hombres quienes generan contenidos digitales y, entre ellos, los más jóvenes y mejor formados.

\section{La competencia digital en el desarrollo profesional}

Algunos estudios han mostrado que la intensidad y heterogeneidad de uso de internet y las tecnologías digitales influye de manera decisiva en la habilidad y autonomía de uso (Hargittai, 2010; Jiménez-Cortés, Rebollo-Catalán, García-Pérez, \& Buzón-García, 2015), mientras que otros apuntan a que son las motivaciones y usos profesionales los responsables en el aumento de la habilidad digital (Castaño, Duart, \& Sancho, 2012; Livingstone \& Helsper, 2007) demostrando que es el hábito de usar la tecnología para trabajar en red e interaccionar con personas lo que favorece la integración en posiciones aventajadas del mercado laboral. De hecho, Navarro (2009) señala que son factores como el nivel educativo, la carrera profesional y el acceso al trabajo los que inciden más directamente en la brecha digital.

Nikitov y Sainty (2014) han mostrado que la inversión en capital social mediante el uso de redes sociales está fuertemente asociada con el éxito profesional, constatando que es la cantidad de actividad con fines profesionales en las redes sociales lo que tiene una fuerte y consistente relación con el éxito profesional; este estudio además muestra que una fuerte inversión en usos personales no solo no marca diferencias, sino que puede influir negativamente en el desarrollo profesional.

Sung, Chi Man Ng, Loke, y Ramos (2013) identifican la resolución de problemas, la competencia emocional y la comunicación efectiva como habilidades clave para la empleabilidad, todas ellas implicadas en el uso de las redes sociales virtuales y otras tecnologías digitales interactivas. Rebollo-Catalán, Jiménez-Cortés y García-Pérez (2016) muestran que las mujeres profesionales tienen una alta percepción de utilidad de las tecnologías digitales, pero incluso entre las gerentes y profesionales altamente cualificadas se observa poca iniciativa e innovación en el uso de éstas.

En su estudio con mujeres profesionales, Kumra y Vinnicombe (2010) demuestran que éstas, siendo conscientes de la necesidad de acumular capital social para avanzar en su desarrollo profesional, utilizan una variedad de estrategias para mejorar su reputación y credibilidad cuando acceden y usan las redes sociales, muchas de ellas encaminadas a minimizar el impacto de ciertos estereotipos que prevalecen en la cultura de las organizaciones laborales tales como el "estereotipo de mujer ambiciosa o disponible".

\section{Método}

\section{Objetivos}

Nuestro objetivo es conocer el grado y tipo de competencia digital que desarrollan las mujeres en el uso de las redes sociales virtuales, con la finalidad de identificar perfiles de mujeres según su habilidad digital en las redes y explorar diferencias en la competencia digital en función de su situación laboral. 


\section{Población y Muestra}

La población está constituida por mujeres adultas de Andalucía y Extremadura que usan redes sociales. La muestra está formada por 1340 mujeres con una experiencia mínima de un año en el uso de redes sociales virtuales, la cual ha sido seleccionada mediante un muestreo estratificado por cuotas, considerando la edad y la situación laboral. La tabla 1 resume los datos sociodemográficos de las participantes.

Tabla 1

Datos sociodemográficos de la muestra

\begin{tabular}{lccc}
\hline Variable & Categoría & Frecuencia & Porcentaje \\
\hline Edad & $18-25$ años & 322 & $24.1 \%$ \\
& $26-34$ años & 218 & $16.3 \%$ \\
& $35-44$ años & 284 & $21.2 \%$ \\
Procedencia geográ- & $45-54$ años & 299 & $22.3 \%$ \\
fica & Ruraños & 215 & $16.1 \%$ \\
Nivel educativo & Urbano & 491 & $36.6 \%$ \\
& Primaria & 849 & $63.4 \%$ \\
& Secundaria & 364 & $27.3 \%$ \\
Situación laboral & Universidad & 504 & $37.8 \%$ \\
& Desempleada & 465 & $34.9 \%$ \\
& Profesional & 458 & $42.5 \%$ \\
& Empresaria & 494 & $45.8 \%$ \\
& & 126 & $11.7 \%$ \\
\hline
\end{tabular}

En cuanto a su perfil tecnológico, encontramos que casi el $80 \%$ de las mujeres utiliza Internet diariamente y casi un $70 \%$ tiene una experiencia de uso superior a 5 años. Más del $80 \%$ de las mujeres utiliza dispositivos móviles para manejar las redes, siendo el móvil y el portátil los más usados. El 86.3\% de ellas lo tiene a su disposición de forma exclusiva. Las mujeres de nuestra muestra hacen un uso variado e intenso de las redes sociales, encontrando que la mayoría usa tres redes sociales o más de manera frecuente. La red de uso más generalizada es WhatsApp, seguida de Facebook y YouTube. La tabla 2 muestra el perfil tecnológico de las mujeres participantes. 
Tabla 2

Descripción de los usos tecnológicos de la muestra

\begin{tabular}{|c|c|c|c|}
\hline Variable & Categoría & Frecuencia & Porcentaje \\
\hline \multirow{4}{*}{$\begin{array}{l}\text { Experiencia de uso } \\
\text { de Internet }\end{array}$} & $<1$ año & 81 & $6.1 \%$ \\
\hline & 1-2 años & 116 & $8.7 \%$ \\
\hline & 2-5 años & 215 & $16.1 \%$ \\
\hline & $>5$ años & 921 & $67.1 \%$ \\
\hline \multirow{3}{*}{$\begin{array}{l}\text { Frecuencia de uso de } \\
\text { Internet }\end{array}$} & Mensual & 107 & $8 \%$ \\
\hline & Semanal & 162 & $12.1 \%$ \\
\hline & A diario & 1065 & $79.8 \%$ \\
\hline \multirow[t]{5}{*}{ Horario de uso } & A todas horas & 375 & $28.5 \%$ \\
\hline & Por la tarde & 137 & $10.4 \%$ \\
\hline & Por la tarde/noche & 473 & $35.9 \%$ \\
\hline & Ocasional & 178 & $13.5 \%$ \\
\hline & Por la mañana/tarde & 153 & $11.6 \%$ \\
\hline \multirow[t]{4}{*}{ Dispositivo de uso } & Ordenador & 163 & $14.3 \%$ \\
\hline & Portátil & 254 & $2.3 \%$ \\
\hline & Móvil & 652 & $57.3 \%$ \\
\hline & Tablet & 45 & $4 \%$ \\
\hline \multirow{2}{*}{$\begin{array}{l}\text { Disponibilidad del } \\
\text { dispositivo }\end{array}$} & Uso exclusivo & 1144 & $86.3 \%$ \\
\hline & Uso compartido & 181 & $13.7 \%$ \\
\hline \multirow{3}{*}{$\begin{array}{l}\text { Uso habitual de re- } \\
\text { des sociales }\end{array}$} & Solo WhatsApp & 268 & $20.1 \%$ \\
\hline & $\begin{array}{c}\text { Además, Facebook y } \\
\text { YouTube }\end{array}$ & 672 & $50.3 \%$ \\
\hline & $\begin{array}{c}\text { Además, Instagram y } \\
\text { Twitter }\end{array}$ & 396 & $29.6 \%$ \\
\hline
\end{tabular}

\section{Instrumento}

La encuesta empleada incluye las siguientes secciones:

a) Características sociodemográficas y de uso de las tecnologías. Se pregunta a las participantes sobre su edad, lugar de residencia, nivel educativo, situación laboral y cuestiones relacionadas con su experiencia de uso de Internet (frecuencia de uso, dispositivos de acceso, redes sociales, etc.) con el propósito de obtener una descripción de la muestra que permita estudiar su posible influencia sobre otras variables. 
b) Escala de competencias digitales en las redes sociales. Incluye una escala elaborada ad hoc a partir de estudios previos (Area \& Pessoa, 2012; García-Pérez, R., Rebollo-Catalán, \& García-Pérez, F.F., 2016; Janssen et al., 2013) que consta de 30 ítems agrupados en cinco categorías que miden las competencias informativas, funcionales, emocionales, de seguridad y creativas, con un rango de respuesta de 0 -nunca- a 3 -siempre-. Aplicando un análisis categórico de componentes principales mediante un procedimiento de escalamiento óptimo para datos ordinales, comprobamos que la medida es fiable $(\alpha=.926)$ y válida (con índices de correlación por encima de .30 de todos los ítems en el componente principal). Todas las subdimensiones de la escala son también fiables (informativa $=.84$; funcional $=.82$; emocional $=.80 ;$ seguridad $=.80$; creativa $=.82$ ). La tabla 3 muestra los coeficientes de correlación (Rho de Spearman-Brown) entre todas las subdimensiones que conforman la escala, encontrándose que todos los coeficientes expresan una muy alta relación. De forma complementaria, con el propósito de reducir el sesgo ocasionado por medidas de autoinforme, aplicamos la escala de autoeficacia percibida en el uso de las TIC de Howard (2014) como medida de validez concurrente (Rho $=.691 ; p \leq .01)$.

Tabla 3

Correlaciones entre las subdimensiones de la escala

\begin{tabular}{|c|c|c|c|c|c|}
\hline & $\begin{array}{c}\text { F1. } \\
\text { Informativa }\end{array}$ & $\begin{array}{c}\text { F2. } \\
\text { Creativa }\end{array}$ & $\begin{array}{c}\text { F3. } \\
\text { Seguridad }\end{array}$ & $\begin{array}{c}\text { F4. } \\
\text { Emocional }\end{array}$ & $\begin{array}{c}\text { F5. } \\
\text { Funcional }\end{array}$ \\
\hline F1. Informativa & 1 & & & & \\
\hline $\begin{array}{c}\text { F2. } \\
\text { Creativa }\end{array}$ & $-656^{* *}$ & 1 & & & \\
\hline $\begin{array}{c}\text { F3. } \\
\text { Seguridad }\end{array}$ & $.606^{* *}$ & $.478^{* *}$ & 1 & & \\
\hline $\begin{array}{c}\text { F4. } \\
\text { Emocional }\end{array}$ & $.536^{* *}$ & $.405^{* *}$ & $.556^{* *}$ & 1 & \\
\hline $\begin{array}{c}\text { F5. } \\
\text { Funcional }\end{array}$ & $.715^{* *}$ & $.610^{* *}$ & $.561^{* *}$ & $.513^{* *}$ & 1 \\
\hline
\end{tabular}

$(* *) p<.001$.

\section{Procedimiento y análisis de datos}

Para el proceso de recogida de la información contamos con la colaboración del Instituto de la Mujer de ambas regiones para dar a conocer la investigación y divulgar la encuesta entre las mujeres. La encuesta se realiza en formato electrónico con Google Docs. Se precisa un consentimiento informado de las mujeres, por lo que se incluye en la encuesta una sección que informa a las mismas sobre el propósito del estudio y la naturaleza voluntaria y anónima de sus respuestas. 
Los datos obtenidos son analizados con el paquete estadístico SPSS (versión 23). Una vez determinada la estructura factorial de la escala de competencias digitales, creamos una variable nueva con la puntuación global a partir del sumatorio de todos sus ítems. Para este trabajo, presentamos los resultados en una escala de tres puntos (0-29 = básica; 30-59 = moderada; y, 60-90 =avanzada), una vez comprobada que dicha recodificación no afecta a su validez y fiabilidad de la medida y que se ajusta a los estándares internacionales de la medida de la habilidad digital. También se crean variables nuevas con el sumatorio de los ítems por cada tipo de competencia (informativa, creativa, seguridad, emocional y funcional).

En relación al tratamiento estadístico de los datos, se realiza un análisis exploratorio de las variables en estudio para conocer su distribución. Asimismo, se aplica un análisis de conglomerados de $\mathrm{K}$ medias (Q-Cluster) para establecer perfiles de mujeres en función de su competencia digital en el uso de las redes sociales. Por último, se aplica un análisis bivariante a través de tablas de contingencia y pruebas de Kruskal-Wallis y Tukey para determinar diferencias en la competencia digital en función al perfil laboral de las mujeres. Aplicamos la d de Cohen para valorar la magnitud de las diferencias.

\section{Resultados}

A continuación, presentamos los resultados obtenidos en el estudio, aludiendo en primer lugar, al grado y tipos de competencia digital de las mujeres en las redes sociales, seguido de los obtenidos en relación a los perfiles de mujeres según su habilidad en el uso de las redes y las diferencias que muestran según su situación laboral.

\section{Grado de competencia digital en las redes sociales}

Respecto al primer objetivo de investigación que se propone conocer el grado de competencia digital de las mujeres en el uso de las redes sociales virtuales, los datos evidencian un nivel medio-alto de competencia digital por parte de las encuestadas $(\mathrm{M}=$ 50.21 ; DT $=18.50$ ), encontrando que el $53.9 \%$ de las mujeres que ya usan las redes sociales se perciben competentes en un nivel moderado y el $32.7 \%$ a nivel avanzado, mientras que sólo el 13.4\% lo hace en un nivel básico. Estos datos sugieren que la mayoría de las mujeres manejan exitosa y eficazmente las redes sociales virtuales, aspecto que puede estar condicionado por el criterio de inclusión del propio estudio, determinado por una experiencia de uso de las redes sociales de al menos un año.

Los resultados del estudio reflejan que las mujeres destacan por la regulación emocional que desarrollan al utilizar las redes sociales $(\mathrm{M}=1.97$; DT $=0.711)$, seguido por las competencias funcionales $(\mathrm{M}=1.96$; $\mathrm{DT}=0.717)$ y la configuración de la privacidad y seguridad en las mismas $(\mathrm{M}=1.82 ; \mathrm{DT}=0.900)$. Encontramos que los aspectos en los que las mujeres puntúan más bajo es en las competencias informativas, es decir, en el manejo y gestión de información a través de las redes sociales $(\mathrm{M}=1.53$; $\mathrm{DT}=0.706)$ $\mathrm{y}$, sobre todo, en la creación de contenidos digitales propios $(\mathrm{M}=1.11$; $\mathrm{DT}=0.737)$. La figura 1 muestra la distribución de mujeres según el nivel de logro autopercibido en cada dimensión competencial. 


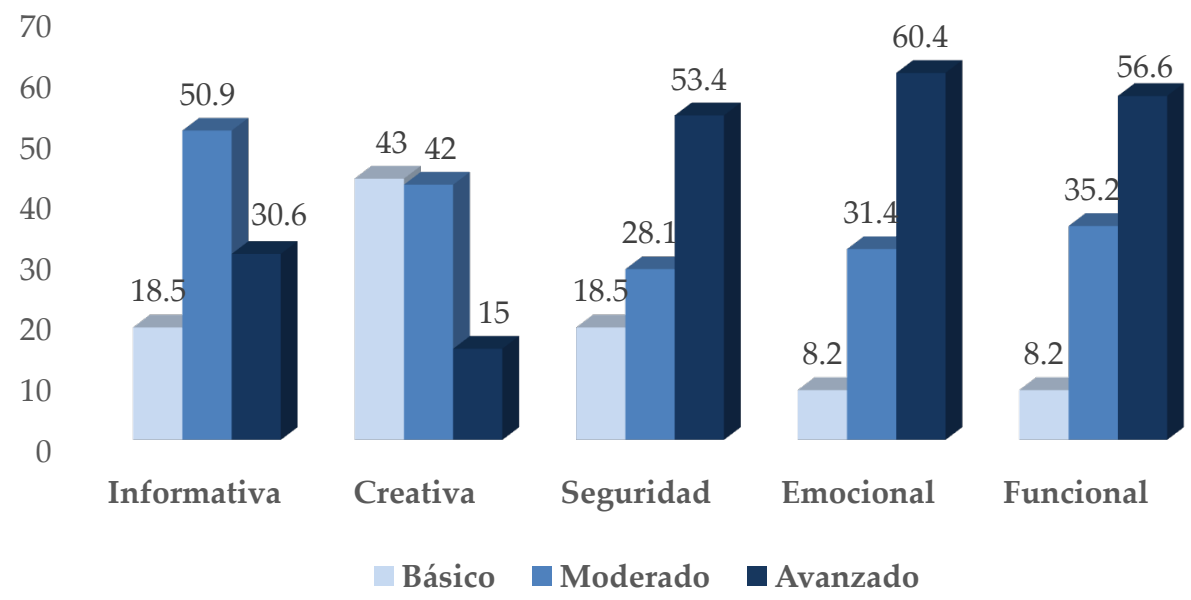

Figura 1. Distribución de mujeres según dimensiones de competencia digital.

\section{Perfiles de mujeres según su competencia digital}

Para el segundo objetivo del estudio, encaminado a identificar perfiles de mujeres según su competencia digital en el uso de las redes sociales, realizamos un análisis de conglomerados clúster de las cinco modalidades de competencia digital, que nos permite identificar cuatro perfiles de mujeres significativamente diferenciados en función a su progresivo uso y dominio de las competencias digitales (tabla 4 y 5).

Tabla 4

Partición (K-medias) en 4 grupos

\begin{tabular}{ccccc}
\hline $\begin{array}{c}\text { Factores } \\
\text { competencia digital }\end{array}$ & 1 & 2 & 3 & \\
\hline & 0.50 & 1.10 & .51 & 2.17 \\
\hline F1. Informativa & 0.37 & 0.68 & 0.89 & 1.87 \\
\hline F2. Creativa & 0.48 & 0.99 & 2.43 & 2.29 \\
\hline F3. Seguridad & 0.51 & 1.83 & 2.17 & 2.32 \\
\hline F4. Emocional & 0.66 & 1.75 & 1.99 & 2.51 \\
\hline F5. Funcional & & &
\end{tabular}


Tabla 5

ANOVA exploratorio e impacto de cada factor

\begin{tabular}{|c|c|c|c|c|c|c|c|c|}
\hline \multirow{2}{*}{$\begin{array}{c} \\
\text { Factores } \\
\text { competencia } \\
\text { digital } \\
\end{array}$} & \multicolumn{6}{|c|}{ ANOVA } & \multirow{2}{*}{\multicolumn{2}{|c|}{ Impacto }} \\
\hline & \multicolumn{2}{|c|}{ Conglomerado } & \multicolumn{2}{|c|}{ Error } & & & & \\
\hline & $\begin{array}{l}\text { Media } \\
\text { cuad. }\end{array}$ & Gl & $\begin{array}{l}\text { Media } \\
\text { cuad. }\end{array}$ & Gl & $\mathrm{F}$ & Sig. & $\begin{array}{c}\text { Tamaño } \\
\text { efecto } \\
\text { dCohen }\end{array}$ & $\mathrm{r}$ \\
\hline $\begin{array}{l}\text { F1. Informa- } \\
\text { tiva }\end{array}$ & 211.353 & 3 & .506 & 1278 & 417.518 & .000 & 3.4 & .863 \\
\hline $\begin{array}{c}\text { F2. } \\
\text { Creativa }\end{array}$ & 213.603 & 3 & .501 & 1278 & 426.412 & .000 & 3.1 & .838 \\
\hline $\begin{array}{l}\text { F3. Seguri- } \\
\text { dad }\end{array}$ & 266.246 & 3 & .377 & 1278 & 705.554 & .000 & 4.1 & .898 \\
\hline $\begin{array}{c}\text { F4. Emocio- } \\
\text { nal }\end{array}$ & 198.259 & 3 & .537 & 1278 & 369.231 & .000 & 3.7 & .878 \\
\hline F5. Funcional & 132.155 & 3 & .692 & 1278 & 190.940 & .000 & 4.0 & .896 \\
\hline
\end{tabular}

Los datos muestran que las mujeres que se incluyen en el primer perfil presentan competencias digitales "deficientes" ( $\mathrm{f}=130 ; \mathrm{P}=10.1$ ), obteniendo puntuaciones muy bajas en todas las habilidades digitales, incluso en las competencias más básicas que implican un uso seguro de la red. El segundo grupo de mujeres cuentan con unas competencias digitales "básicas" ( $\mathrm{f}=329 ; \mathrm{P}=25.7)$, destacando en habilidades de comunicación y regulación emocional al utilizar las redes sociales. No obstante, este grupo obtiene bajas puntuaciones en las competencias relacionadas con la selección y gestión de la información y, sobre todo, con la creación de contenidos digitales propios. El tercer perfil de mujeres muestra competencias digitales "moderadas" en el uso de las redes sociales $(\mathrm{f}=382 ; \mathrm{P}=29.8$ ), destacando por habilidades de seguridad y privacidad, pero con pocas habilidades emocionales y funcionales de las herramientas que ponen a su disposición las redes. El último grupo de mujeres presenta el nivel más elevado en el manejo de habilidades digitales, por lo que ha sido denominado de carácter "avanzado" $(\mathrm{f}=441 ; \mathrm{P}=34.4)$. Este perfil destaca sobre los anteriores en la selección y gestión de información a través de la red y en la creación de contenidos digitales propios (imagen, texto, vídeo, información, etc.), además de obtener excelentes puntuaciones en el uso funcional de las herramientas que poseen las redes, y en lo referido a la seguridad y al manejo emocional en las mismas. La figura 2 muestra la distribución de estos perfiles en la muestra analizada. 


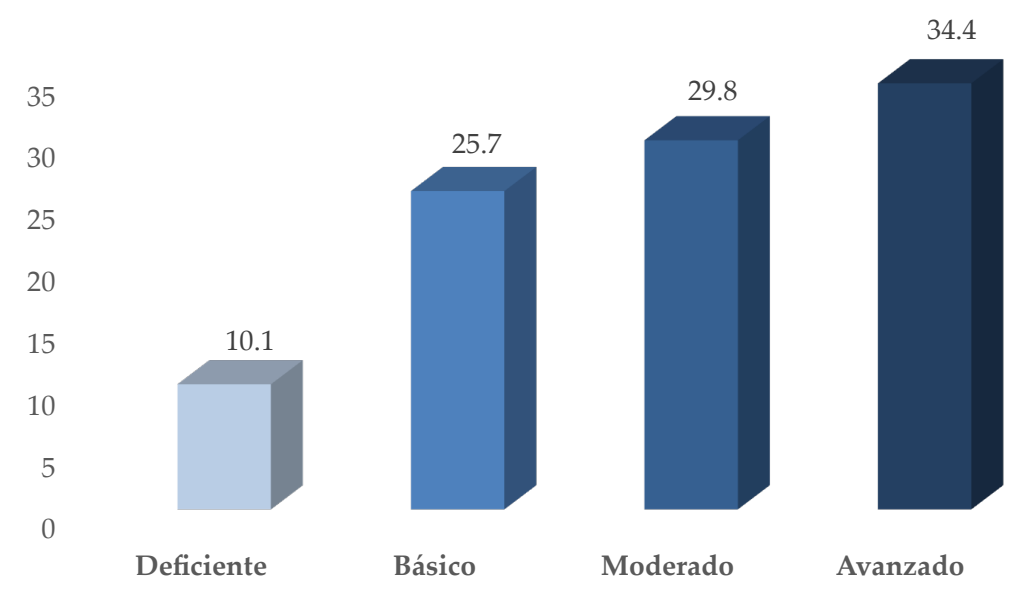

Figura 2. Distribución de mujeres por nivel de competencia digital.

Los resultados muestran que el perfil de mujeres con competencias digitales avanzadas es el que alcanza un nivel de competencia en el uso de las redes sociales más elevado $(\mathrm{M}=66.90$; $\mathrm{DT}=9.66)$, estando un $34.4 \%$ de las encuestadas en este grupo $(\mathrm{f}=441)$. Le siguen las mujeres que presentan el nivel de competencia digital moderado $(\mathrm{M}=52.42$; DT $=8.42$ ), en el que se sitúan casi el $29.8 \%$ de las participantes del estudio $(\mathrm{f}=382$ ). Encontramos que el $25.7 \%$ presenta capacidades básicas de uso y dominio de las redes sociales virtuales $(\mathrm{M}=39.12 ; \mathrm{DT}=8.95 ; \mathrm{f}=329) \mathrm{y}$, por último, encontramos un grupo de mujeres que muestran niveles deficientes de competencia $(\mathrm{M}=15.22$; $\mathrm{DT}=12.30)$, estando en este perfil un $10 \%$, una de las encuestadas ( $\mathrm{f}=130)$. La figura 3 ilustra las diferencias entre estos perfiles de mujeres en función de su nivel de competencia digital.

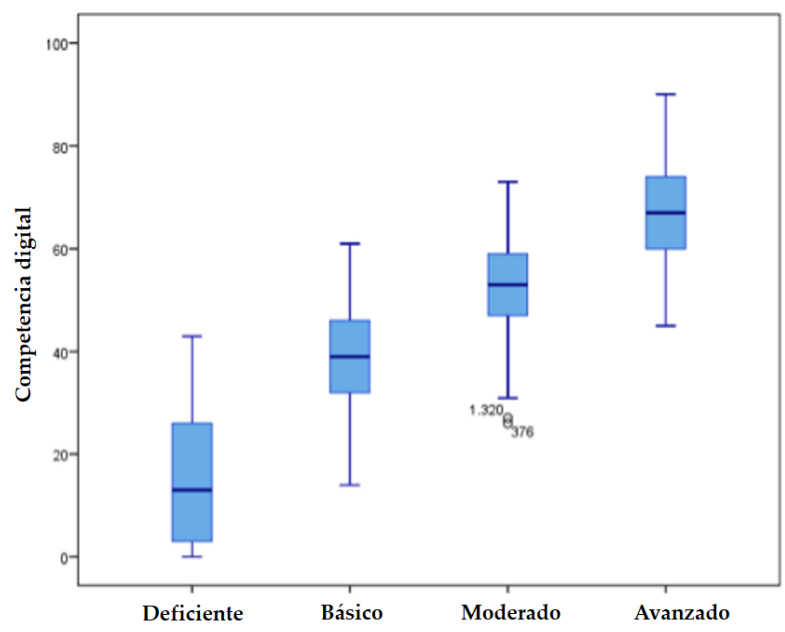

Figura 3. Nivel de competencia digital en función de perfiles de mujeres. 


\section{Diferencias en la competencia digital según situación laboral}

Con respecto al último objetivo de investigación, que pretende analizar las diferencias en la competencia digital según situación laboral de las mujeres, los resultados muestran diferencias significativas en la competencia digital en función de su situación laboral $\left(\chi^{2}=23.928 ; p<.001 ; \mathrm{C}\right.$. Contingencia $\left.=.150\right)$. La tabla 6 muestra los resultados del coeficiente de contingencia y chi-cuadrado en la variable competencia digital en función de la situación laboral de las mujeres.

Tabla 6

Tabla de contingencia de la competencia digital en función de la situación laboral

\begin{tabular}{|c|c|c|c|c|c|}
\hline & & \multicolumn{3}{|c|}{ Perfil laboral } & \multirow[b]{2}{*}{ Sig } \\
\hline & & $\begin{array}{c}\text { Desempleada } \\
\quad(n=446)\end{array}$ & $\begin{array}{c}\text { Profesional } \\
(\mathrm{n}=474)\end{array}$ & $\begin{array}{l}\text { Empresaria } \\
(n=122)\end{array}$ & \\
\hline \multirow{6}{*}{$\begin{array}{c}\text { Nivel } \\
\text { competencia } \\
\text { digital }\end{array}$} & & 136 & 116 & 37 & \multirow{6}{*}{$\begin{array}{c}\chi^{2}=23,928 \\
p=.001\end{array}$} \\
\hline & Deficiente & $(30.5 \%)$ & $(24.5 \%)$ & $(30.3 \%)$ & \\
\hline & Básico & $\begin{array}{c}110 \\
(24.7 \%)\end{array}$ & $\begin{array}{c}144 \\
(30.4 \%)\end{array}$ & $\begin{array}{c}39 \\
(32.0 \%)\end{array}$ & \\
\hline & & 131 & 176 & 36 & \\
\hline & Moderado & $(29.4 \%)$ & $(37.1 \%)$ & & \\
\hline & Avanzado & $\begin{array}{c}136 \\
(30.5 \%)\end{array}$ & $\begin{array}{c}116 \\
(24.5 \%)\end{array}$ & $\begin{array}{c}37 \\
(30.3 \%)\end{array}$ & \\
\hline
\end{tabular}

Aplicado el Anova de Kruskal-Wallis, encontramos que las diferencias observadas en el nivel de competencia digital en el uso de las redes sociales en función del perfil laboral de las mujeres son estadísticamente significativas tanto globalmente $\left(\chi^{2}=13.583\right.$; $p<.01)$ como por dimensión competencial: competencias informativas $\left(\chi^{2}=18.447 ; p\right.$ $<.01)$, de seguridad $\left(\chi^{2}=16.039 ; p<.01\right)$, emocionales $\left(\chi^{2}=10.044 ; p<.01\right)$ y creativas $\left(\chi^{2}=8.482 ; p<.01\right)$. Unicamente no se observan diferencias en las competencias funcionales $\left(\chi^{2}=4.856 ; p>.05\right)$.

La prueba ANOVA de Tukey (tabla 7) nos permite comprobar que son las mujeres profesionales las que presentan niveles más elevados de competencia digital $(\mathrm{M}=51.60$; $\mathrm{DT}=17.62)$, seguidas de las mujeres empresarias $(\mathrm{M}=48.38$; $\mathrm{DT}=15.41)$. En contraste, el perfil de las mujeres que se encuentran en situación de desempleo es el que presenta competencias digitales más bajas en el uso de las redes sociales $(\mathrm{M}=46.76$; $\mathrm{DT}=20.02)$. Por tanto, encontramos que los perfiles entre los que se observan mayores diferencias son los de mujeres desempleadas y profesionales (figura 4). 
Tabla 7

Prueba post hoc de Tukey de competencia digital en función de perfiles laborales

\begin{tabular}{cccc}
\hline & & \multicolumn{2}{c}{ Subconjunto para alfa $=\mathbf{0 , 0 5}$} \\
\cline { 3 - 4 } Perfil laboral & $\mathbf{1}$ & $\mathbf{1}$ & $\mathbf{2}$ \\
\hline Desempleada & 446 & 46.74 & \\
\hline Empresaria & 122 & 48.38 & 48.38 \\
\hline Profesional & 474 & & 51.60 \\
\hline Sig. & & .602 & .136 \\
\hline
\end{tabular}

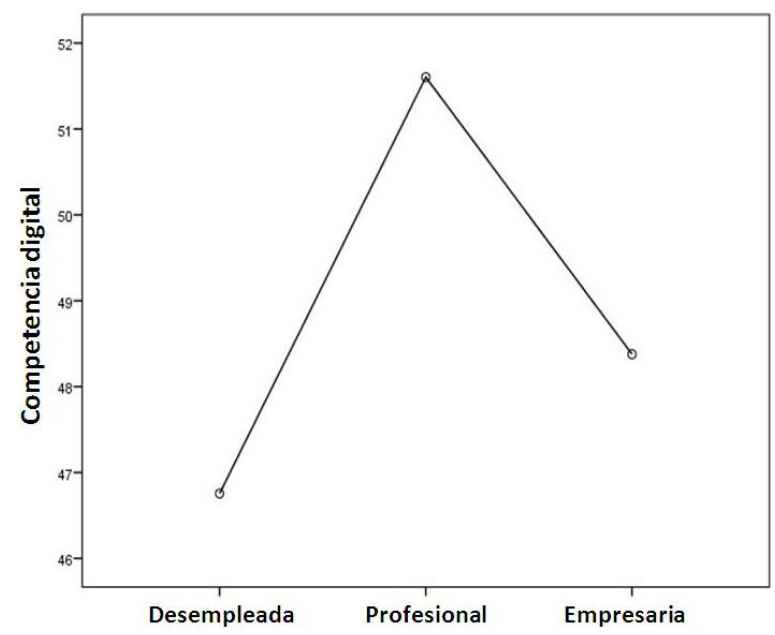

Figura 4. Gráfico de medias de la competencia digital y perfiles laborales de las mujeres.

\section{Discusión y conclusiones}

Con esta investigación hemos querido analizar el logro de competencias digitales en las redes sociales virtuales en mujeres que ya las usan, dado que el Plan de Inclusión Digital y Empleabilidad se fija como objetivo aumentar las competencias TIC en mujeres que ya usan tecnologías digitales (MIET, 2013). La aparente reducción de la brecha digital de género en el uso de las redes sociales (Clipson, Wilson, \& DuFrene, 2012), así como su valor estratégico para aumentar el capital social y la empleabilidad (Castaño et al., 2012; Nikitov \& Sainty 2014; Vega-Caro et al., 2015) son motivos suficientes para centrar el estudio específicamente en esta tecnología.

Los resultados de este estudio han mostrado que la mayor parte de las mujeres encuestadas se perciben con un nivel medio-alto de competencias digitales en el uso de 
las redes sociales, destacando especialmente en competencias emocionales, funcionales y de seguridad en las redes, mientras que presentan deficiencias en competencias informativas y creativas. Estos resultados son coincidentes con los de investigaciones previas (García-Ruiz et al., 2014; Lee et al., 2015; Sánchez-Carrero \& Contreras-Pulido, 2012) que han mostrado que la creación de contenidos digitales continua siendo una asignatura pendiente. Algunas de estas investigaciones (Pérez-Escoda \& RodríguezConde, 2016) han documentado que incluso en un colectivo como el profesorado faltan competencias digitales en un nivel suficiente que les permita innovar en su actividad docente. Este dato resulta de interés para diseñar una formación en TIC ajustada a las necesidades y perfil de las mujeres centrada en la creación de contenidos digitales, pero también en la búsqueda, manejo y gestión de información útil y vinculada a usos concretos. Algunos investigadores (Gómez-Torres, 2016; González-Oñatu, Fanjul-Peyró, \& Cabezuelo-Lorenzo, 2015) han advertido de las repercusiones que la falta de conocimiento y destrezas relacionadas con la búsqueda de información en la sociedad digital tiene en la integración socio-laboral.

Los resultados obtenidos en relación a los grupos de mujeres según su competencia digital en las redes sociales son coincidentes con los de investigaciones internacionales previas que han determinado niveles o grados de inclusión digital en función de la experiencia, intensidad y habilidad de uso de Internet y otras tecnologías digitales (Hargittai, 2010; Livingstone \& Helsper, 2007). Esta investigación muestra que aún entre mujeres adultas con una experiencia mínima de un año en el uso de las redes sociales podemos encontrar un tercio de mujeres que no tienen un nivel de destreza suficiente que les permita hacer un uso eficiente de las mismas, siendo las competencias funcionales y emocionales las más transversales, ya que en todos los grupos identificados de mujeres las desarrollan en un nivel mínimo, lo que puede deberse a la propia naturaleza interactiva y social de esta tecnología.

Por último, los resultados muestran diferencias en el nivel de competencia digital autopercibida de las mujeres en función de su situación laboral, encontrando que son las profesionales y empresarias las que obtienen competencias digitales más avanzadas en el uso de las redes sociales respecto a las desempleadas. Al haber controlado el posible sesgo derivado de medidas de autoinforme incorporando una medida adicional para la validación concurrente como es la autoeficacia percibida en el uso de tecnologías (Howard, 2014), podemos afirmar que estos resultados no se encuentran sesgados por el efecto que la autopercepción de eficacia según situación laboral puede tener en la competencia digital en las redes sociales.

Los resultados también indican que estas diferencias no solo se observan en la competencia digital global sino en todos los tipos de competencias excepto en las funcionales que se refieren a habilidades instrumentales y comunicativas básicas. Sung et al. (2013) identificó las habilidades emocionales, comunicativas y de resolución de problemas como habilidades básicas para la empleabilidad. Nuestros datos parecen indicar que estas habilidades las tienen desarrolladas a nivel básico las mujeres con carácter general en relación con el uso de las redes sociales, sin embargo otras facetas que parecen clave en el desarrollo profesional no. Como señala Navarro (2009) el acceso al trabajo es uno de los factores que más inciden en la brecha digital. Los resultados de nuestro estudio confirman esto, al mostrar al colectivo de desemplea- 
das como el que mayores necesidades de formación presenta, no solo en tipos de competencias adquiridas sino también en nivel de destreza alcanzado. Esto revela la urgencia de una formación en el uso de las redes sociales virtuales orientada a mujeres desempleadas para la búsqueda activa de empleo para aumentar sus competencias para la empleabilidad.

El conocimiento generado con esta investigación permite adecuar mejor la formación a las mujeres según el perfil y necesidades que presenta. Mientras que las mujeres desempleadas necesitan aumentar su capital social cara a su empleabilidad, lo que pone especial énfasis en las habilidades informativas, las mujeres profesionales y, más aún, las empresarias, necesitan aumentar sus habilidades para la creación y difusión de contenidos digitales que aumenten su visibilidad y mejoren su reputación. No obstante, queremos señalar la necesidad de continuar profundizando en la competencia digital de las mujeres considerando su perfil y situación laboral, incorporando otras variables que pueden aportar claves para el diseño de la formación como son los motivos de uso (Castaño et al., 2012; Jiménez-Cortés et al., 2015; Nikitov \& Sainty, 2014) pero también creemos junto con otras investigadoras (Kumra \& Vinnicombe, 2010; Rubio \& Escofet, 2013; Vergés, 2012) que para lograr la autoinclusión y empoderamiento de las mujeres en TIC es necesario incorporar una mirada en la investigación sobre los contextos de uso y las formas de interacción por las cuales se consolidan ciertos valores y prácticas culturales.

\section{Referencias}

Area, M., \& Pessoa, T. (2012). De lo sólido a lo líquido: Las nuevas alfabetizaciones ante los cambios culturales de la Web 2.0. Comunicar, 19(38), 13-20. doi: http:// dx.doi.org/10.3916/C38-2012-02-01

Braun, M.T. (2013). Obstacles to social networking website use among older adults. Computers in Human Behavior, 29, 673-680. doi: http://dx.doi.org/10.1016/j. chb.2012.12.004

Castaño, C., Martin, J., \& Vázquez, S. (2008). La e-inclusión y el bienestar social: una perspectiva de género. Economía Industrial, 367, 139-152.

Castaño, J., Duart, J.M., \& Sancho, T. (2012). Una segunda brecha digital entre el alumnado universitario. Cultura y Educación, 24(3), 363-377.

Clipson, T.W., Wilson, S.A., \& DuFrene, D.D. (2012). The Social Networking Arena: Battle of the Sexes. Business Communication Quarterly, 75(1), 64-67.

Comisión Europea (2013). Europa 2020: la estrategia europea de crecimiento. Luxemburgo: Oficina de Publicaciones de la Unión Europea. Recuperado de: http://ec.europa. eu/europe2020/index_es.htm

Dias, I. (2012). O uso das tecnologías digitais entre os seniores. Motivações e intereses. Sociologia, Problemas e Práticas, 68, 51-77. doi: http://dx.doi.org/10.7458/ SPP201268693

García-Pérez, R., Rebollo-Catalán, A., \& García-Pérez, F.F. (2016). Relación entre las preferencias de formación del profesorado y su competencia digital en las redes sociales. Bordón. Revista de pedagogía, 68(2), 137-153. doi: http://dx.doi.org/10.13042/ Bordon.2016.68209 
García-Pérez, R., Mayor-Buzon, V., \& Rebollo-Catalán, A. (2016). La alfabetización digital de las mujeres en las redes sociales. En R. Jiménez-Cortes, A. RebolloCatalán, \& R. García-Pérez (Coords.), Aprendizaje con tecnologías digitales para la e-inclusión (pp. 55-66). Madrid: Síntesis.

García-Ruiz, R., Ramírez-García, A., \& Rodríguez-Rosell, M.M. (2014). Educación en alfabetización mediática para una nueva ciudadanía prosumidora. Comunicar, 43, 15-24. doi: http://dx.doi.org/10.3916/C43-2014-01

Gómez-Torres, M.J. (2016). El papel de la alfabetización digital en la Empleabilidad de los trabajadores mayores. Pixel-bit. Revista de Medios y Educación, 49, 25-38. doi: http://dx.doi.org/10.12795/pixelbit.2016.i49.02

González-Oñate, C., Fanjul-Peyró, C., \& Cabezuelo-Lorenzo, F. (2015). Uso, consumo y conocimiento de las nuevas tecnologías en personas mayores en Francia, Reino Unido y España. Comunicar, 45, 19-28. doi: http://dx.doi.org/10.3916/C45-2015-02

Hargittai, E., \& Shafer, S. (2006). Differences in Actual and Perceived Online Skills: The Role of Gender. Social Science Quarterly, 87(2), 432-448. doi: http:/dx.doi. org/10.1111/j.1540-6237.2006.00389.x

Hargittai, E. (2010). Digital Natives? Variation in Internet Skills and Uses among Members of the "Net Generation". Sociological Inquiry, 80(1), 92-113. doi: http:// dx.doi.org/10.1111/j.1475-682X.2009.00317.x

Howard, M.C. (2014). Creation of a computer self-efficacy measure: analysis of internal consistency, psychometric properties, and validity. Cyberpsychology, behavior, and social networking, 17(10), 677-681. doi: http://dx.doi.org/10.1089/cyber.2014.0255

Janssen, J., Stoyanov, S., Ferrari, A., Punie, Y., Pannekeet, K., \& Sloep, P. (2013). Experts' views on digital competence: Commonalities and differences. Computers $\mathcal{E}$ Education, 68, 473-481. doi: http://dx.doi.org/10.1016/j.compedu.2013.06.008

Jiménez-Cortés, R., Rebollo-Catalán, A., García-Pérez, R., \& Buzón-García, O. (2015). Motivos de uso de las redes sociales virtuales: Análisis de perfiles de mujeres rurales. RELIEVE, Revista Electrónica de Investigación y Evaluación Educativa, 21(1), 1-17. doi: http://dx.doi.org/10.7203/relieve.21.1.5153

Kumra, S., \& Vinnicombe, S. (2010). Impressing for success: A gendered analysis of a key social capital accumulation strategy. Gender, Work $\mathcal{E}$ Organization, 17(5), 521-546.

Lee, L., Chen, D.-T., Li, J.-Y., \& Lin, T.-B. (2015). Understanding new media literacy: The development of a measuring instrument. Computers $\mathcal{E}$ Education, 85, 84-93. doi: http://dx.doi.org/10.1016/j.compedu.2015.02.006

Lin, C., Tang, W., \& Kuo, F. (2012). Mommy Wants to Learn the Computer: How MiddleAged and Elderly Women in Taiwan Learn ICT Through Social Support. Adult Education Quarterly, 62(1), 73-90. doi: http://dx.doi.org/10.1177/0741713610392760.

Livingstone, S., \& Helsper, E. (2007). Gradations in digital inclusion: children, young people and the digital divide. New Media E Society, 9(4), 671-696. doi: http://dx.doi. org/10.1177/1461444807080335

Luna, H., Mendoza, R., \& Álvarez, F.J. (2015). Patrones de diseño para mejorar la accesibilidad y uso de las aplicaciones sociales para adultos mayores. Comunicar, 45, 85-94. doi: http://dx.doi.org/10.3916/C45-2015-09. 
MIET (2013). Plan de Inclusión Digital y Empleabilidad. Ministerio de Industria, Economía y Turismo, Gobierno de España: Madrid. Recuperado de: http://www. agendadigital.gob.es/planes-actuaciones/Bibliotecainclusion/Detalle\%20del\%20 Plan/Plan-ADpE-7_Inclusion-Empleabilidad.pdf

Navarro, M. (2009). La brecha digital de género en España: cambios y persistencias. Feminismo/s, 14, 183-200.

Nikitov, A., \& Sainty, B. (2014). The role of social media in influencing career success. International Journal of Accounting \& Information Management, 22(4), 273-294. doi: http://dx.doi.org/10.1108/IJAIM-02-2014-0009

Pérez-Escoda, A., \& Rodríguez-Conde, M.J. (2016). Evaluación de las competencias digitales autopercibidas del profesorado del profesorado de Educación Primaria en Castilla y León. Revista de Investigación Educativa, 34(2), 399-415. doi: http:// dx.doi.org/10.6018/rie.34.2.215121

Rebollo-Catalán, A., Jiménez-Cortés, R., \& García-Pérez, R. (2016). El aprendizaje de mujeres profesionales en las redes sociales. En R. Jiménez-Cortes, A. RebolloCatalán \& R. García-Pérez (Coords.), Aprendizaje con tecnologías digitales para la e-inclusión (pp. 147-162). Madrid: Síntesis.

Rebollo-Catalán, A., Vico-Bosch, A., \& García-Pérez, R. (2015). El aprendizaje de las mujeres de las redes sociales y su incidencia en la competencia digital. Prisma Social: Revista de Ciencias Sociales, 15, 122-146.

Rubio, M.J., \& Escofet, A. (2013). Estudio sobre los usos de las TIC y las posibilidades de empoderamiento en las mujeres. Revista Iberoamericana de Educación, 62(3), 1-13.

Sánchez-Carrero, J., \& Contreras-Pulido, P. (2012). De cara al prosumidor: producción y consumo empoderando a la ciudadanía 3.0. ICONO14, 10(3), 62-84. doi: http:// dx.doi.org/10.7195/ri14.v10i3.210

Sánchez-Vadillo, N., Ortega, O., \& Vall-Llovera, M. (2012). Romper la brecha digital de género. Factores implicados en la opción por una carrera tecnológica. Athenea Digital, 12(3), 115-128. doi: http://dx.doi.org/10.5565/rev/athenead/v12n3.1133

Sung, J., Chi Man Ng, M., Loke, F., \& Ramos, C. (2013). The nature of employability skills: empirical evidence from Singapore. International Journal of Training and Development, 17(3), 176-193. doi: http://dx.doi.org/10.1111/ijtd.12008

Torrent-Sellens, J. (2014). Aprendizaje virtual, trabajo en red y salarios: ¿nueva empleabilidad, nuevas paradojas? Oikonomics. Revista de Economía, Empresa y Sociedad, 1, 65-71.

Vega-Caro, L., Vico-Bosch, A., \& Rebollo-Catalán, A. (2015). Motivaciones de uso de las redes sociales para el desarrollo del capital social de las mujeres de entorno rural. ICONO14, 13(2), 142-162. doi: http://dx.doi.org/10.7195/ri14.v13i2.839

Vekiri, I., \& Chronaki, A. (2008). Gender issues in technology use: Perceived social support, computer self-efficacy and value beliefs, and computer use beyond school. Computers \& Education, 51(3), 1392-1404. doi: http://dx.doi.org/10.1016/j. compedu.2008.01.003

Vergés, N. (2012). De la exclusión a la autoinclusión de las mujeres en las TIC. Motivaciones, posibilitadores y mecanismos de autoinclusión. Athenea Digital, 12(3), 129-150. 
Wajcman, J. (2012). TIC e inequidad ¿ganancias en red para las mujeres? Revista Educación y Pedagogía, 24(62), 117-134. Recuperado de http://aprendeenlinea. udea.edu.co/revistas/index.php/revistaeyp/article/view/14198/12541

Watson, W. R., Watson, S. L., \& Reigeluth, C. M. (2015). Education 3.0: breaking the mold with technology. Interactive Learning Environments, 23(3), 332-343. doi: http:// dx.doi.org/10.1080/10494820.2013.764322

Fecha de recepción: 11 de octubre de 2016

Fecha de revisión: 11 de octubre de 2016

Fecha de aceptación: 27 de marzo de 2017 